\title{
Impacto do Programa Academia da Saúde sobre gastos com internações hospitalares por doenças cerebrovasculares
}

\author{
Impact of the Health Gym Program on hospital admissions for cerebrovascular diseases
}

\section{AUTORES}

Rita de Cássia Franciele Lima ${ }^{1,3}$ (iD

Bárbara Letícia Silvestre Rodrigues ${ }^{3}$ (D) Shirlley Jackllanny Martins de Farias ${ }^{3}$ (D) Bruno Rodrigo da Silva Lippo ${ }^{2}$ (D) Flávio Renato Barros da Guarda ${ }^{3}$ (D)

1 Secretária Municipal de Saúde de Jaboatão dos Guararapes, Jaboatão dos Guararapes, Pernambuco, Brasil.

2 Universidade Federal de Pernambuco, Departamento de Educação Física, Recife,

Pernambuco, Brasil.

3 Universidade Federal de Pernambuco, Centro Acadêmico de Vitória, Vitória de Santo Antão, Pernambuco, Brasil.

\section{CONTATO}

Rita de Cássia Franciele Lima

cassia_franciele@hotmail.com

Rua Santa Flora, N²90, apartamento 104, Prazeres, Jaboatão dos Guararapes, Pernambuco, Brasil.

CEP: 54310-180.

DOI

$10.12820 /$ rbafs. $25 \mathrm{e} 0166$

\section{(cc) BY}

Este trabalho está licenciado com uma Licença Creative Commons - Atribuição 4.0 Internacional.

\begin{abstract}
RESUMO
O objetivo deste artigo é avaliar o impacto do Programa Academia da Saúde (PAS) sobre os gastos com internações hospitalares por doenças cerebrovasculares no estado de Pernambuco. Trata-se de uma avaliação de impacto de políticas públicas, desenvolvida através de uma abordagem quase-experimental que consiste na aplicação do método do pareamento por escore de propensão, tomando como referência os anos de 2010 e 2018. Para tanto, utilizou-se dados socioeconômicos, demográficos e epidemiológicos de 89 municípios que implantaram o programa (tratados) e de outros 52 que não implantaram (controles). Os dados foram obtidos no Departamento de Informática do Sistema Único de Saúde, Instituto Brasileiro de Geografia e Estatística e outras bases. O total de internações por doenças cerebrovasculares em 2010 foi de 6.091 e 10.595 em 2018. Os municípios que implantaram o PAS gastaram em média $\mathrm{R} \$ 1.258,61$ a menos com internações por doenças cerebrovasculares $(\mathrm{p}<0,05)$ para cada grupo de 10 mil habitantes. O modelo econométrico proposto mostrou-se adequado para explicar o impacto do PAS sobre os gastos com internações hospitalares por doenças cerebrovasculares. A relação entre a implantação PAS e a diminuição do gasto nos permite inferir que essa intervenção tem cumprido com a sua diretriz de constituir-se como programa de referência para a promoção da saúde, prevenção e controle de doenças crônicas, e alcançado o seu objetivo específico de aumentar o nível de atividade física da população dos municípios beneficiários.
\end{abstract}

Palavras-chave: Doenças cerebrovasculares; Hospitalizações; Política de saúde; Avaliação de programas e projetos de saúde; Avaliação do impacto na saúde.

\begin{abstract}
Health Gym Program (HGP) is a strategic health and physical activity promotion program financed by the Brazilian public health system. The aim of this paper is to analyze the impact of HGP on the spending on hospital admissions for cardiovascular disease in Pernambuco State, Brazil. This public policy impact analysis had used a quasi-experimental approach which consists on the application of the Propensity Score Matching considering the years 2010 and 2018. Socioeconomics, demographics and epidemiological data of 89 municipalities that implemented HGP (treated) and 54 that did not (controls) were collected from Brazilian Health Data Department, Brazilian Institute of Geography and Statistics and other databases. The total number of hospitalizations for cerebrovascular diseases was 6,091 in 2010 and 10,595 in 2018. Municipalities that implemented HGP spent an average of $R \$ 1,258.61$ less on hospitalizations for Cerebrovascular Disease $(p<0.05)$ for each group of 10,000 inhabitants. The econometric model developed in this study is adequate to explain the impact of the HGP on the expenses with hospital admissions for cerebrovascular diseases. The relationship between HGP implantation and the decrease in spending on hospital admissions allows us to infer that this intervention has complied with its guideline of constituting itself as a reference program for the health promotion, prevention and control of chronic diseases, and achieved its specific objective of increasing the level of physical activity of the population of the beneficiary municipalities.
\end{abstract}

Keywords: Cerebrovascular disease; Hospitalization; Health Policy; Program evaluation; Health impact assessment.

\section{Introdução}

O enfrentamento das Doenças Crônicas não Transmissíveis (DCNT) é um dos principais desafios de saúde pública no mundo. No Brasil, as DCNT são as mais prevalentes, responsáveis em 2011, por $68,3 \%$ do total de mortes ${ }^{1,2}$. Cabe ressaltar que as DCNT interferem na qualidade de vida e na produtividade dos indivíduos, aumentando o risco de morte prematura, e contribuindo para o aumento nos gastos do Sistema Único de Saúde (SUS), por serem doenças de longa duração e demandarem maior quantidade de ações, procedimentos e serviços de saúde, por períodos de tempo prolongados ${ }^{3}$. 
Dentre as DCNT mais prevalentes destacam-se as doenças cardiovasculares $(30,4 \%)$, as neoplasias $(16,4 \%)$, as doenças respiratórias $(6,0 \%)$ e o diabetes, com $5,3 \%^{1}$, o que caracteriza um importante desafio para os gestores de saúde no sentido da qualificação das ações e das respostas às demandas dos portadores dessas doenças ${ }^{2}$.

No ano de 2010 ocorreram 326 mil mortes por doenças cardiovasculares, das quais 200 mil deveram-se exclusivamente à doença isquêmica do coração e às doenças cerebrovasculares $(\mathrm{DCBV})^{4}$. As Doenças Cerebrovasculares (DCBV) estão em segundo lugar entre as doenças com maior mortalidade no mundo, e as estimativas apontam que essa posição tende a se manter, pelo menos até o ano de $2030^{5}$.

As DCBV são mais prevalentes em pessoas menos escolarizadas e em municípios com piores indicadores de desigualdade (índice de Gini) ${ }^{6}$. Elas foram responsáveis por 179.054 internações hospitalares no Brasil em 2018, o que demandou um gasto de $\mathrm{R} \$ 268.321 .647,00$ para o Sistema Único de Saúde ${ }^{7}$.

Os principais fatores de risco para as DCBV são de natureza comportamental (tabagismo, hábitos alimentares, consumo nocivo de álcool e inatividade física) ${ }^{8}$, com destaque para a inatividade física, a qual aumenta o risco de desenvolvimento dessas doenças 9 .

Por outro lado, evidências apontam que a prática regular de atividade física reduz em $26 \%$ o risco de desenvolvimento e mortalidade por AVC, quando comparados os indivíduos ativos com indivíduos pouco ati$\operatorname{vos}^{10}$. Além disso, uma diminuição de $10 \%$ no número de indivíduos fisicamente inativos no Brasil geraria uma economia de $\mathrm{R} \$ 24.081 .636,89$ com internações hospitalares por doenças relacionadas à inatividade física ${ }^{11}$, o que tem reforçado a importância da adoção e políticas e programas públicos com foco no incentivo à prática regular de atividades físicas em diferentes grupos populacionais.

Entre as políticas brasileiras de estímulo à adoção de estilos de vida mais ativos e saudáveis destaca-se a criação do Programa Academia da Saúde (PAS), instituído em 2011, e que foi desenvolvido para figurar como um equipamento da rede de serviços que visa potencializar ações de cuidados específicos, individuais e coletivos com o foco na promoção da saúde e na produção do cuidado ${ }^{12,13}$.

O PAS tem como objetivo geral contribuir para a promoção da saúde através da criação de polos com infraestrutura e pessoal qualificado para desenvolver ações de promoção da saúde e produção do cuidado no contexto da atenção primária à saúde no âmbito dos municípios ${ }^{12,13}$.

O programa desempenha um papel fundamental na Atenção Primária, configurando-se como uma estratégia importante para a melhoria da qualidade de vida da população e para integração de propostas de ação previstas nas políticas nacionais de Atenção Básica e de Promoção da Saúde, além de compor o escopo das atividades previstas no Plano de Ações Estratégicas para o Enfrentamento das $\mathrm{DCNT}^{14}$, configurando-se como a estratégia oficial de incentivo à prática de atividades físicas contidas nesse plano ${ }^{9,13}$.

Embora o PAS possua um amplo escopo de objetivos específicos voltados à promoção da saúde e adoção de modos de vida saudáveis ${ }^{12}$, evidências apontam que o objetivo específico mais trabalhado nos municípios é o que visa aumentar o nível de atividade física da população ${ }^{13}$.

Segundo Guarda et al..$^{15}$, a implantação do PAS no estado de Pernambuco ocorreu em uma velocidade maior que a da maioria das demais Unidades de $\mathrm{Fe}-$ deração, pois o processo de implementação dos polos do PAS permitia a incorporação de programas de AF já existentes nos municípios, desde que esses tivessem características similares às do PAS. Nesse sentido, entre 2011 e 2014 foram implementados 2.538 polos do PAS nos estados brasileiros, sendo que 266 desses polos $(10,48 \%)$ encontram-se implantados no estado de Pernambuco ${ }^{16}$.

Apesar de alguns estudos apontarem que o PAS é efetivo para aumentar o nível de atividade física da população ${ }^{17,18}$, e de um grande conjunto de evidências apontar que a prática regular de atividades físicas pode diminuir a morbimortalidade por $\mathrm{DCBV}^{19}$, ainda são limitados os estudos que investigaram o impacto de programas de promoção da saúde e da AF sobre os gastos públicos em saúde no contexto geral ${ }^{20}$ e sobre os gastos com DCBV de modo específico.

Neste sentido, o objetivo do presente estudo é avaliar o impacto do Programa Academia da Saúde sobre os gastos com internações hospitalares por DCBV no estado de Pernambuco.

\section{Método}

Esta avaliação de impacto de políticas públicas foi desenvolvida através de uma abordagem quase-experimental que consiste na aplicação do método do pareamento por escore de propensão (aqui designado como Propensity Score Matching - PSM) para estimar o efeito 
médio do tratamento sobre os tratados (ATT). O ATT é caracterizado neste estudo pelo efeito do Programa Academia da Saúde sobre os gastos com internações hospitalares por doenças cerebrovasculares no estado de Pernambuco.

O grupo de tratados é composto pelos 89 municípios pernambucanos que implantaram o Programa Academia da Saúde no ano de 2011 e os controles são as 52 cidades que não implantaram. Foram excluídos da amostra os municípios que implantaram o programa após o ano de 2011 e os que o implantaram nesse ano e cancelaram as suas atividades nos anos subsequentes. Também foi excluído da amostra o município de Recife, pois apresenta o programa Academia da Cidade desde 2002, que se assemelha a proposta do Programa Academia da Saúde e, portanto, poderia enviesar a avaliação.

Neste estudo foram consideradas as seguintes doenças cerebrovasculares: Hemorragia Intracraniana, Infarto Cerebral, Acidente Vascular Não-Especificado Como Hemorrágico ou Isquêmico e Outras DCBV. A escolha se deve ao fato de que um conjunto de evidências robustas aponta a associação entre a inatividade física e o risco de adoecimento e morte por essas doen$\operatorname{ças}^{20}$ e pelo fato de que o PAS tem como um dos seus objetivos específicos o aumento do nível de atividade física da população ${ }^{13}$.

Os dados foram coletados tomando como referência os anos de 2010 (ano anterior à implantação) e 2018 (sete anos após o início da implantação do PAS no estado de Pernambuco).

O estudo utilizou dados secundários de domínio público que foram obtidos no Departamento de Informática do SUS (DATASUS) oriundos do Sistema de Informações Hospitalares, do Cadastro Nacional de Estabelecimentos de Saúde e do Departamento de Atenção Básica. Os dados foram extraídos com auxílio da ferramenta TABnet do próprio DATASUS. Para as causas estudadas, foram consideradas as seguintes doenças cerebrovasculares: Hemorragia Intracerebral, Infarto Cerebral, Acidente Vascular Cerebral Não-Especificado como hemorrágico ou isquêmico e "Outras Doenças Cerebrovasculares" conforme última Classificação Internacional das Doenças, 10a Revisão (CID-10).

Os gastos com internações hospitalares por DCBV consideram o total de Autorizações de Internações Hospitalares (AIH) pagas, tomando como referência o município de residência do indivíduo.

Quanto aos dados socioeconômicos e demográficos dos municípios, esses foram obtidos no site do Insti- tuto Brasileiro de Geografia e Estatística (IBGE); da Base de Dados do Estado, e; do Índice FIRJAN de Desenvolvimento Municipal (IFDM), da Federação das Indústrias do Estado do Rio de Janeiro (FIRJAN).

A variável de desfecho utilizada é a taxa média de gasto com internações hospitalares por DCBV para cada 10 mil habitantes. As variáveis que compõem a análise foram escolhidas de acordo com os modelos epidemiológicos que indicam os fatores associados às internações hospitalares (na população brasileira) por doenças crônicas não-transmissíveis de forma geral, e por doenças cerebrovasculares de forma específica ${ }^{21}$.

Dessa forma, foi utilizado um conjunto de covariáveis que constitui aspectos socioeconômicos, demográficos epidemiológicos e de acesso a serviços de saúde. Entre elas estão: População geral por município; Índice FIRJAN de desenvolvimento Municipal (IFDM); Índice FIRJAN de desenvolvimento relacionado à saúde, à educação e ao emprego e renda (separados por áreas); cobertura da Atenção Básica dos municípios; proporção da população feminina e masculina; Produto Interno Bruto (PIB) per Capita; Índice de Desenvolvimento Humano (IDH); taxa de leitos hospitalares para cada 1.000 habitantes; a proporção de residentes por cada faixa etária (40 a 49 anos, 50 a 59 anos, 60 a 69 anos, 70 a 79 anos e de 80 anos e mais), número de óbitos gerais, número de óbitos por hipertensão arterial, número de óbitos relacionados a hipertensão arterial por sexo, faixa etária e raça/cor.

Foram adotados procedimentos de estatística descritiva (frequências, médias e desvios-padrão) para caracterizar o perfil socioeconômico, demográfico e epidemiológico dos municípios tratados e controles antes do pareamento. Para o cálculo das diferenças entre as médias dos expostos e não expostos à política em análise (Programa Academia da Saúde), foi utilizado a medida D' Choens e para as proporções foi utilizado o teste exato de Fisher.

As análises relacionadas ao método de pareamento por escore de propensão serão descritas a seguir. Elas foram realizadas no software estatístico STATA e os resultados apresentados em gráficos e tabelas.

De acordo com o referencial normativo do programa, a implantação do PAS foi facultada aos municípios que quisessem incorporar essa intervenção ao conjunto de ações da atenção primária à saúde ${ }^{12,15}$. Nesse sentido, considerando que não houve aleatoriedade na composição dos grupos expostos e não expostos a essa intervenção, e a amostra que compõe este estudo poderia estar sujeita a problemas como o viés de seleção, e sensível à 
multidimensionalidade dos fatores que determinam a probabilidade de implantação dessa política ${ }^{22}$, utilizou-se o método do pareamento por escore de propensão (Propensity Score Matching - PSM), permitindo constituir um grupo de controle com características observáveis semelhantes às do grupo exposto à intervenção (tratados) ${ }^{23}$.

O PSM permite a formação de dois grupos em relação algumas características socioeconômicas, demográficas e epidemiológicas e calcula a probabilidade de os municípios aderirem ao programa com base nesses perfis, criando um cenário contrafactual que permite comparar os municípios tratados e controles ${ }^{22,23}$.

Os procedimentos de pareamento utilizam um escore balanceado, computado a partir de um modelo de regressão (logit ou probit) que utiliza uma variável dependente.

Selecionados os tratados e controles, a probabilidade de os municípios implantarem o PAS foi estimada através do modelo logit que considerou um vetor de características do período anterior à exposição ao programa ${ }^{24}$, o qual é dado por:

$$
\operatorname{Pr}\left(P \mathrm{AS}_{i, 0}=1\right)=\varnothing\left(\beta X_{i},-1\right)
$$

onde $\varnothing$ é uma função de distribuição acumulada logística e $\mathrm{PAS}_{\mathrm{i}}$ é uma variável dummy que assume o valor 1 se o município é tratado (exposto ao PAS) e 0 caso não seja tratado. A realização do pareamento por variáveis referentes ao período anterior à criação do programa se justifica pela grande adesão de municípios pernambucanos ao PAS no ano de 2011, sobretudo por conta do disposto na Portaria Ministerial no 1.402/2011, a qual permitia que municípios que possuíssem intervenções com características e espaços físicos similares aos do PAS pudessem imediatamente aderir ao programa ${ }^{14,15}$. Dessa forma, as características dos municípios podem ter sido afetadas pela intervenção já no ano inicial do programa, o que poderia causar uma distorção nos resultados da análise, caso fossem inseridas as cidades que implantaram o programa nos anos subsequentes.

A probabilidade de o município ser tratado, dado o conjunto de caraterísticas $X$, é denominada de escore de propensão:

$$
\hat{\mathrm{P}}(\mathrm{X})=\operatorname{Pr}\left(\operatorname{PAS}_{i, 0}=1 \mid X_{i},-1\right)
$$

Para calcular o escore de propensão foram selecionadas variáveis que descreveram as características dos municípios que potencialmente explicam a sua adesão para implantar o PAS.
$\mathrm{Na}$ etapa seguinte, os escores de propensão estimados foram utilizados para computar os pesos necessários para balancear os municípios no grupo de controle, de modo que, em sua média, esses se tornem semelhantes aos tratados. Para tanto, foram testados os métodos do vizinho mais próximo (1:5), com e sem reposição, pareamento de Kernel e pareamento radial, de modo a identificar a melhor forma de realizar o pareamento ${ }^{11}$.

Para testar a qualidade do ajuste e a robustez do modelo, foi elaborado um gráfico de ajustamento, o qual permite verificar a distribuição de probabilidade estimada pelo PSM.

Todas as análises foram realizadas no software Stata versão 15.0, considerando o delineamento complexo da amostra. Utilizou-se o aplicativo do Stata "psmatch2" para o cálculo do propensity score e do ATT, adotando um nível de significância de 5\% para todos os testes estatísticos.

\section{Resultados}

Foram registradas 6.091 internações por DCBV no ano de 2010 e 10.595 no ano de 2018 em Pernambuco. Essas correspondem, respectivamente, a 1,18\% e 1,92\% do total de internações, por todas as doenças em 2010 e 2018 no estado.

A Tabela 1 descreve as médias e os desvios-padrão das variáveis que compuseram os modelos de análise do impacto do PAS, de acordo com os grupos tratado e controle, antes do pareamento. Observou-se que municípios tratados apresentavam em 2010 melhores indicadores de PIB, IDH, Taxa de desemprego, Taxa de Analfabetismo e Índice de desenvolvimento municipal relacionado à educação (FIRJAN Educação).

Para realizar o pareamento por escore de propensão foram estimados modelos logit e probit, considerando as variáveis socioeconômicas, demográficas e da rede de atenção à saúde dos municípios que compõe a amostra. $\mathrm{O}$ modelo logit mostrou-se mais adequando e com melhor significância estatística, e foi escolhido para avaliar o impacto do PAS sobre o gasto com internações hospitalares por DCBV. Os resultados do modelo logit são apresentados na Tabela 2, juntamente com os testes dos algoritmos de pareamento.

As variáveis que promoveram o melhor ajuste do modelo foram a Cobertura da Atenção Básica, os Índices de Firjan relacionados à educação e à saúde, a taxa de desemprego e o PIB per capita.

A construção dos escores de propensão baseados na regressão logit retornou os coeficientes mostrados. 
Tabela 1 - Médias e desvios-padrão das características epidemiológicas, socioeconômicas e relacionadas a rede de atenção à saúde nos munícipios. Pernambuco, 2010 e 2018.

\begin{tabular}{|c|c|c|c|c|c|c|c|c|c|c|c|}
\hline \multirow{3}{*}{ Variáveis } & \multicolumn{4}{|c|}{2010} & \multicolumn{4}{|c|}{2018} & \multirow{3}{*}{ d-Cohen } & \multirow{3}{*}{\multicolumn{2}{|c|}{ IC 95\% }} \\
\hline & \multicolumn{2}{|c|}{ Controles } & \multicolumn{2}{|c|}{ Tratados } & \multicolumn{2}{|c|}{ Controles } & \multicolumn{2}{|c|}{ Tratados } & & & \\
\hline & Média & DP & Média & DP & Média & DP & Média & $\mathrm{DP}$ & & & \\
\hline Prop homens & 0,508 & 0,008 & 0,505 & 0,008 & 0,494 & 0,008 & 0,487 & 0,041 & 0,243 & 0,0014 & 0,4871 \\
\hline Prop mulheres & 0,512 & 0,008 & 0,516 & 0,008 & 0,505 & 0,008 & 0,504 & 0,043 & 0,021 & 0,2223 & 0,2646 \\
\hline Prop 40 a 49 anos & 0,109 & 0,013 & 0,111 & 0,013 & 0,109 & 0,014 & 0,111 & 0,013 & 0,157 & 0,0861 & 0,4015 \\
\hline Prop 50 a 59 anos & 0,076 & 0,007 & 0,078 & 0,010 & 0,076 & 0,007 & 0,078 & 0,010 & 0,214 & 0,0298 & 0,4583 \\
\hline Prop 60 a 69 anos & 0,055 & 0,010 & 0,059 & 0,010 & 0,055 & 0,010 & 0,059 & 0,009 & 0,397 & 0,1518 & 0,6432 \\
\hline Prop 70 a 79 anos & 0,033 & 0,008 & 0,035 & 0,008 & 0,033 & 0,008 & 0,035 & 0,008 & 0,274 & 0,0296 & 0,5187 \\
\hline Prop 80 anos e mais & 0,016 & 0,006 & 0,018 & 0,005 & 0,016 & 0,005 & 0,018 & 0,005 & 0,358 & 0,1133 & 0,6038 \\
\hline PIB & $5.967,01^{*}$ & $2.858,54$ & $6.549,09^{*}$ & $4.208,75$ & $9.821,88^{*}$ & $5.879,93$ & $10.704,86^{*}$ & $6.514,10$ & 0,175 & 0,0684 & 0,4194 \\
\hline IDH & 0,588 & 0,044 & 0,602 & 0,048 & 0,587 & 0,047 & 0,602 & 0,047 & 0,302 & 0,0572 & 0,5467 \\
\hline Taxa Analfabetismo & 27,09 & 6,88 & 25,46 & 7,59 & 27,21 & 7,25 & 25,54 & 7,37 & $-0,225$ & $-0,4692$ & 0,0192 \\
\hline Taxa Desemprego & 10,36 & 5,32 & 8,19 & 3,70 & 10,40 & 5,39 & 8,35 & 3,85 & $-0,479$ & $-0,7258$ & $-0,2324$ \\
\hline Cob Atenção Básica & 0,92 & 0,140 & 0,85 & 0,194 & 0,91 & 0,16 & 0,92 & 0,130 & $-0,152$ & $-0,3957$ & 0,0918 \\
\hline FIRJAN Educação & 0,577 & 0,067 & 0,604 & 0,064 & 0,655 & 0,065 & 0,700 & 0,069 & 0,502 & 0,2555 & 0,7495 \\
\hline FIRJAN Saúde & 0,642 & 0,107 & 0,622 & 0,125 & 0,807 & 0,051 & 0,803 & 0,083 & $-0,022$ & $-0,2662$ & 0,2207 \\
\hline
\end{tabular}

*Valores em Reais; IC 95\%* = Intervalo de Confiança 95\% da medida d de Cohen ; PIB = Produto Interno Bruto; IDH = Índice de Desenvolvimento Humano; FIRJAN = Federação das Indústrias do Estado do Rio de Janeiro.

Tabela 2-Média dos gastos por Doenças Cerebrovasculares nos municípios que implantaram e que não implantaram polos do Programa Academia da Saúde. Pernambuco, 2010 e 2018.

\begin{tabular}{|c|c|c|c|c|c|c|c|c|c|c|c|}
\hline \multirow{3}{*}{ Variáveis } & \multicolumn{4}{|c|}{2010} & \multicolumn{4}{|c|}{2018} & \multirow{3}{*}{ D-Cohens } & \multirow{2}{*}{\multicolumn{2}{|c|}{ IC $95 \%$}} \\
\hline & \multicolumn{2}{|c|}{ Tratados } & \multicolumn{2}{|c|}{ Controles } & \multicolumn{2}{|c|}{ Tratados } & \multicolumn{2}{|c|}{ Controles } & & & \\
\hline & Média & DP & Média & DP & Média & DP & Média & DP & & & \\
\hline $\begin{array}{l}\text { Acidente Vascular } \\
\text { Cerebral }\end{array}$ & $13.324,26^{*}$ & $42.579,78$ & $7.127,66^{*}$ & $13.457,37$ & $80.575,50 *$ & $180.849,7$ & $41.904,70^{*}$ & $43.147,99$ & 0,223 & $-0,0204$ & 0,4679 \\
\hline $\begin{array}{l}\text { Hemorragia } \\
\text { Intracraniana }\end{array}$ & $22.463,7^{*}$ & $65.303,58$ & $13.210,30^{*}$ & $20.766,27$ & $15.500,28^{*}$ & $31.406,31$ & $7.445,47^{*}$ & $11.565,74$ & 0,197 & -.0471 & 0,4409 \\
\hline Infarto Cerebral & $390,85^{*}$ & $2.126,94$ & $87,79^{*}$ & 355,45 & $5.982,39^{*}$ & $19.875,86$ & $3.319,02^{*}$ & $5.066,71$ & 0,144 & -.0997 & 0,3878 \\
\hline $\begin{array}{l}\text { Outras Doenças } \\
\text { Cerebrovasculares }\end{array}$ & $9.215,19^{*}$ & $34.286,59$ & $2.839,68^{*}$ & $6.995,57$ & $8.560,25^{*}$ & $2.4584,15$ & $2.075,44^{*}$ & $5.957,69$ & 0,264 & 0,0201 & 0,5090 \\
\hline Total DCBV & $45.394,01^{*}$ & $110.601,60$ & $23.265,45^{*}$ & $33.497,98$ & $110.618,4^{*}$ & $249.389,70$ & $54.744,64^{*}$ & $56.934,13$ & 0,257 & 0,0126 & 0,5015 \\
\hline
\end{tabular}

*Valores em Reais; IC 95\%* = Intervalo de Confiança 95\% da medida d de Cohen.

Considerando-se o nível de significância estatística de $5 \%$, os coeficientes estimados revelam que os municípios mais propensos a participar do PAS são aqueles com as menores taxas de desemprego, menor cobertura da Atenção básica e menor índice de desenvolvimento relacionado à saúde (FIRJAN saúde) - Tabela 3.

$\mathrm{Na}$ Tabela 3, são apresentados os impactos estimados do PAS sobre os gastos com internações hospitalares com DCBV, por meio de diferentes algoritmos de pareamento utilizados no escore de propensão. Destaca-se que o pareamento radial mostrou-se mais adequado para explicar o impacto do PAS sobre os gastos com internações hospitalares por doenças cerebro- vasculares ao nível de significância de 5\%, indicando que os municípios que implantaram o PAS gastaram em média, para cada grupo de 10 mil habitantes, $\mathrm{R} \$$ $1.258,61$ a menos com internações por DCBV que as cidades que não implantaram essa intervenção.

Ao analisar a Figura 1, é possível observar a distribuição de probabilidade antes e depois do pareamento dos dois grupos (tratado e controle), avaliando a qualidade do pareamento por escore de propensão. Quanto mais parecidas forem as distribuições após o pareamento, melhor será a capacidade desse de identificar municípios com características semelhantes.

A Figura 1 indica que no momento antes do trata- 
Tabela 3 - Modelo Logit de participação no Programa Academia da Saúde. Pernambuco, 2010 e 2018.

\begin{tabular}{lccccc}
\hline \multicolumn{5}{c}{ Modelo Logit } \\
\hline PAS & Coef. & Erro-Padrão & P>|z & \multicolumn{2}{c}{ IC 95\% } \\
PIB & 0,000 & 0,000 & 0,067 & -3.17 & 0,000 \\
Cobertura AB & $-1,117$ & 0,774 & 0,149 & -2.635 & 0,399 \\
IFDM Educação & 7,778 & 1,637 & 0,000 & 4,569 & 10,987 \\
Taxa desemprego & $-0,104$ & 0,025 & 0,000 & $-0,154$ & $-0,054$ \\
IFDM Saúde & $-2,329$ & 1,090 & 0,033 & $-4,465$ & $-0,192$ \\
Constante & $-1,065$ & 1,100 & 0,333 & $-3,222$ & 1,090 \\
\hline
\end{tabular}

PAS = Programa Academia da Saúde; PIB = Produto Interno Bruto; IFDM = Índice FIRJAN de Desenvolvimento Municipal.

Tabela 4 - Impactos estimados do PAS sobre gastos por AIH com internações hospitalares por DCBV, através do método pareamento por escore de propensão. Pernambuco, 2010 e 2018.

\begin{tabular}{lccc}
\hline \multicolumn{4}{c}{ Gasto por AIH com Doenças Cerebrovasculares (Em Reais) } \\
\hline Algoritmo & Tratado & Controle & Diferença \\
\hline Vizinho mais próximo & $13.536,82$ & $14.051,25$ & $-514,42^{* *}$ \\
Sem algoritmo & $13.536,82$ & $12.973,38$ & $563,44^{* *}$ \\
Kernel & $13.536,82$ & $13.694,67$ & $-157,85^{* *}$ \\
Radial & $13.536,82$ & $14.795,44$ & $-1.258,61^{*}$ \\
\hline
\end{tabular}

$\mathrm{T}=$ * Significante ao nível de 5\% ; * Significante ao nível de $10 \%$.
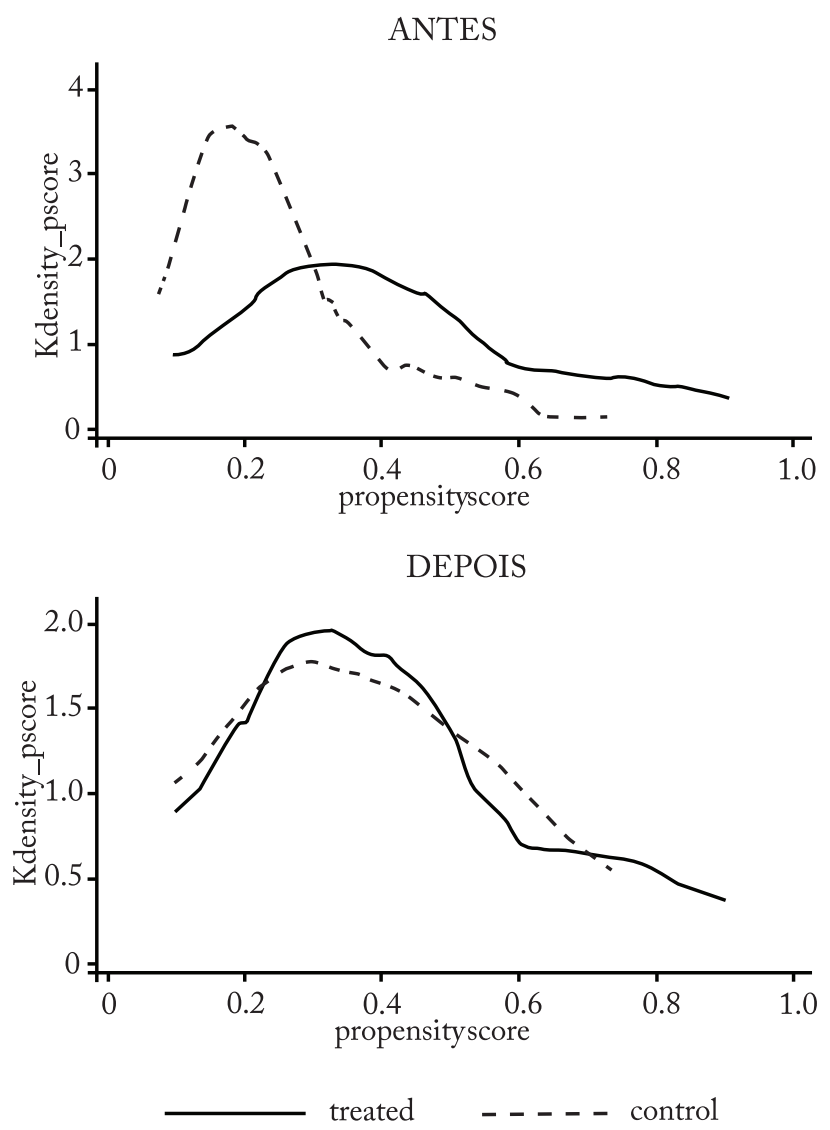

Figura 1 - Distribuição da probabilidade de tratamento antes e depois do pareamento mento (2010) os municípios tratados eram distintos dos controles em características observáveis, com fortes concentrações desses últimos na cauda à esquerda da distribuição. Entretanto, após o pareamento, a distribuição de probabilidade estimada tornou-se intermediariamente semelhante entre tratados e controles, evidenciando uma boa qualidade do matching, e indicando que o modelo proposto permite realizar estimativas mais seguras para o efeito médio do tratamento nos tratados (ATT).

\section{Discussão}

O número de internações por doenças cerebrovasculares em Pernambuco aumentou entre 2011 e 2018, corroborando resultados de outros estudos que apontam um aumento da prevalência dessas doenças em outros estados do Brasil ${ }^{25}$.

Os melhores indicadores de educação, PIB, IDH e taxa de desemprego observados nos municípios tratados antes do pareamento reiteram os achados de estudos que apontam a associação entre fatores socioeconômicos, demográficos e epidemiológicos e maiores prevalências de doenças ${ }^{6}$. Ressalta-se, entretanto, que as diferenças observadas no perfil dos municípios antes do PSM foram eliminadas através da seleção de pares de tratados e controles que apresentassem características e probabilidades de implementação do PAS semelhantes ${ }^{23}$.

No que se refere à estimação do modelo de avaliação do impacto do PAS sobre os gastos com internações hospitalares por DCBV, foram testados modelos do tipo probit e logit, sendo este último o escolhido para mensurar a probabilidade de um município implantar o PAS. O modelo logit é o estimador paramétrico mais amplamente utilizado em pesquisas no campo da avaliação de impacto de políticas públicas ${ }^{26}$. Além disso, se mostrou mais ajustado e apresentou maior significância estatística que o modelo probit.

As variáveis que compuseram o modelo econométrico (Cobertura da Atenção Básica, os Índices de Firjan relacionados à educação e à saúde, a taxa de desemprego e o PIB per capita) corroboram com achados da literatura que relatam a influência da renda, estrutura da rede de atenção à saúde e escolaridade sobre a ocorrência de doenças do aparelho circulatório (inclusive as cerebrovasculares ${ }^{6}$. Além disso, o modelo proposto atendeu às propriedades de balanceamento, as quais indicam que o PSM consegui equilibrar as características observáveis dos grupos tratado e controle ${ }^{22,27}$.

Os resultados dos testes dos algoritmos de pareamento mostraram que o método radial apresentou 
melhores resultados que os demais algoritmos, tanto para o impacto do PAS sobre os gastos com internações por DCBV, quanto na significância estatística do efeito do tratamento sobre os tratados (ATT). O pareamento através do algoritmo radial tornou a probabilidade condicional de implantar polos do PAS mais semelhantes entre os grupos tratado e controle, minimizando o viés de seleção e indicando que o PSM foi bem sucedido ${ }^{27,28}$, o que pode ser verificado por meio da análise visual, a qual indica a presença de uma área de sobreposição das probabilidades condicionais e reforça a presença de uma área de suporte comum entre tratados e controles ${ }^{29}$.

O modelo econométrico estimado neste estudo demonstrou que os municípios que implantaram o PAS tiveram um gasto médio com internações hospitalares por DCBV para cada 10 mil habitantes acima de $\mathrm{R} \$$ $1.200,00$, quando comparados com as cidades que não aderiram a essa intervenção. A relação entre a presença do PAS e a diminuição do gasto pode estar relacionada à adoção de estilos de vida mais ativos por parte da população dos municípios beneficiários, o que nos permite inferir que o objetivo específico do PAS de aumentar o nível de atividade física da população ${ }^{12}$ e a sua diretriz de configurar-se como estratégia de promoção da saúde, produção do cuidado e prevenção de doenças crônicas ${ }^{30}$ está sendo alcançado nos municípios que o implantaram. Entretanto, a falta de metas claramente definidas e de protocolos de ações já relatadas em outros estudos sobre o PAS ${ }^{12,15}$, podem ampliar excessivamente as possibilidades de intervenção e eventualmente comprometer o efeito do programa.

O modelo econométrico desenvolvido neste estudo foi capaz de estabelecer uma boa seleção de grupos de municípios expostos e não expostos ao PAS, utilizou variáveis que corroboram as características socioeconômicas e epidemiológicas que explicam a ocorrência de internações hospitalares por doenças do aparelho circulatório na população brasileira e mostrou-se estatisticamente adequado para explicar o impacto do PAS sobre os gastos com internações hospitalares por doenças cerebrovasculares.

Como limitação deste estudo, destaca-se a impossibilidade de realizar a análise com dados individuados e a não testagem do modelo em estratos populacionais de sexo, faixa etária e raça/cor.

Por outro lado, o presente estudo configura-se como inovador, pois utiliza um método amplamente empregado para a avaliação de impacto de políticas sociais, mas que ainda é pouco explorado no campo da saúde. Além disso, as evidências geradas a partir deste estudo podem servir de referência para avaliar o impacto de outros programas de promoção da saúde e da atividade física e subsidiar a tomada de decisão dos gestores públicos no planejamento de estratégias direcionadas à implantação ou expansão do Programa Academia da Saúde em seus municípios.

\section{Conflito de interesse}

Os autores declaram não haver conflito de interesse.

\section{Contribuição dos autores}

Lima RCF, participou da elaboração do projeto, da revisão da literatura, da redação e revisão do manuscrito. Rodrigues BLS, participou da revisão da literatura e da revisão do manuscrito. Farias SJM, sugeriu elementos da revisão da literatura e revisou o texto. Lippo BRS, sugeriu elementos da revisão da literatura e revisou o texto. Guarda FRB, orientou a elaboração do projeto, sugeriu elementos da revisão da literatura e revisou o texto.

\section{Agradecimentos}

Os autores agradecem ao Grupo de Estudos em Políticas de Saúde, Esporte e Lazer (GEPSEL) pelo apoio e colaboração com a pesquisa.

\section{Referências}

1. GBD 2015 Mortality and Causes of Death Collaborators. Global, regional, and national life expectancy, all-cause mortality, and cause-specific mortality for 249 causes of death, 1980-2015: a systematic analysis for the Global Burden of Disease Study 2015. Lancet 2016;388(10053):1459-544.

2. Duncan BB, Chor D, Aquino EML, Bensenor IM, Mill JG, Schmidt MI, et al. Doenças crônicas não transmissíveis no Brasil: prioridade para enfrentamento e investigação. Rev. Saúde Pública. 2012;46(Suppl1):126-34.

3. Malta DC, Bernal RTI, Lima MG, Araújo SSC, Silva MMA, Freitas MIF et al. Doenças crônicas não transmissíveis e a utilização de serviços de saúde: análise da Pesquisa Nacional de Saúde no Brasil. Rev. Saúde Pública. 2017;51(Suppl 1):4s.

4. Simão AF, Précoma DB, Andrade JP, Correa FH, Saraiva JFK, Oliveira GMM. I Diretriz de Prevenção Cardiovascular da Sociedade Brasileira de Cardiologia - Resumo Executivo. Arq. Bras. Cardiol. 2014 May;102(5):420-31.

5. Brasil. Ministério da Saúde. Secretaria de Atenção à Saúde. Departamento de Atenção Especializada. Manual de rotinas para atenção ao AVC - Brasília, 2013.

6. Figueiredo FSF, Rodrigues TFCS, Rêgo AS, Andrade L, Oliveira RR, Radovanovic CAT.Distribuição e autocorrelação espacial das internações por doenças cardiovasculares em adultos no Brasil. Rev. Gaúcha Enferm. 2020;41:e20190314.

7. Barreto IJB, Guarda FRB, Silva PBC, Silva RN, Farias SJM, Silva AEA. Gastos com internações hospitalares por doenças relacionadas à inatividade física no Brasil. Lecturas: Educación Física y Deportes. 2020;25(265):29-43. 
8. Kaiser SE. Aspectos epidemiológicos nas doenças coronariana e cerebrovascular. Sociedade Brasileira de Cardiologia. Revista da SOCERJ. 2004;17(1):11-5.

9. World Health Organization. The global burden of disease: 2004 update. Geneva: World Health Organization, 2009.

10. Kyu HH, Bachman VF, Alexander LT, Munford JE, Afshin A, Estep K. Physical activity and risk of breast cancer, colon cancer, diabetes, ischemic heart disease, and ischemic stroke events: systematic review and dose-response meta-analysis for the Global Burden of Disease Study 2013. 2016; 354: i3857.

11. Bielemann RM, Silva BGC, Coll CVN, Xavier MO, Silva SG. Impacto da inatividade física e custos de hospitalização por doenças crônicas. Rev. Saúde Públ. 2015;49:75.

12. Silva RN, Guarda FRB, Hallal PC, Martelli PJL. Avaliabilidade do Programa Academia da Saúde no Município do Recife, Pernambuco, Brasil. Cad. Saúde Pública. 2017; 33(4):e00159415.

13. Sá GBAR, Dornelles GC, Cruz KG, Amorim RCA, Andrade SSCA, Oliveira TP, et al. O Programa Academia da Saúde como estratégia de promoção da saúde e modos de vida saudáveis: cenário nacional de implementação. Cien Saude Colet. 2016;21(6):1849-60.

14. Malta DC, Silva Jr JB. O Plano de Ações Estratégicas para o Enfrentamento das Doenças Crônicas Não Transmissíveis no Brasil e a definição das metas globais para o enfrentamento dessas doenças até 2025: uma revisão. Epidemiol. Serv. Saúde. 2013;22(1): 151-64.

15. Guarda F, Silva R, Feitosa W, Santos Neto P, Araújo Júnior J. Caracterização das equipes do Programa Academia da Saúde e do seu processo de trabalho. Rev Bras Ativ Fís Saúde. 2015;20(6):638-40.

16. Departamento de Informática do SUS - Datasus. Cadastro Nacional dos Estabelecimentos de Saúde. [citado em 2020 jul 25]. Disponível em: https://datasus.saude.gov.br/cnesestabelecimentos/.

17. Simoes EJ, Hallal P, Pratt M, Ramos L, Munk M, Damascena W, et al. Effects of a community-based, professionally supervised intervention on physical activity levels among residents of Recife, Brazil. Am J Public Health. 2009;99(1):68-75.

18. Fernandes AP, Andrade ACS, Costa DAS, Dias MAS, Malta DC, Caiaffa WT. Programa Academias da Saúde e a promoção da atividade física na cidade: a experiência de Belo Horizonte, MG, Brasil. Ciênc. saúde coletiva. 2017;22(12):3903-14.
19. Simoes TFP, Ferreira AJ, Martins JC, Faria CDCM. Nível de atividade física de usuários da atenção primária: comparação entre indivíduos saudáveis e pós acidente vascular cerebral. Acta Fisiátr. 2017;24(2):56-61.

20. Abu-Omar K, Rütten A, Burlacu I, Schätzlein V, Messing S, Suhrcke M. O custo-efetividade das intervenções de atividade física: Uma revisão sistemática de revisões. Anterior Rep. Méd. 2017;8:72-8.

21. Lentsck MH, Latorre MRDO, Mathias TAF. Tendência das internações por doenças cardiovasculares sensíveis à atenção primária. Rev Bras epidemiol. 2015;18(2):372-84.

22. Austin,Peter C. An Introduction to Propensity Score Methods for Reducing the Effects of Confounding in Observational Studies. Multivariate behave res. 2011;46(3):399-424.

23. Khandker SR, Koolwal GB, Samad HA. Handbook on Impact Evaluation: Quantitative Methods and Practices. The World Bank. 2010.

24. Fontes LFC, Conceição OC, Saraiva MV. Três anos do programa mais médicos: uma análise econométrica. UFSM. 2016.

25. Lentsck MH, Saito AC, Mathias TAF. tendência de declínio das hospitalizações por doenças cardiovasculares sensíveis à atenção primária. Texto contexto enferm. 2017; 26(2):e03170015.

26. Becker SO, Ichino A. Estimation of average treatment effects based on propensity scores. The Stata Journal, College Station. 2002;2(4):358-77.

27. Ravaioli PF. O impacto do programa Bolsa Família na posse de bens duráveis. [Dissertação de Mestrado]. Piracicaba: Universidade de São Paulo, Escola Superior de Agricultura "Luiz de Queiroz";2018.

28. Paul RR, Donald BR. The central role of the propensity score in observational studies for causal effects, Biometrika, 1983;70(1):41-55.

29. Gertler PJ, Sebastian M, Patrick P, Laura BR, Christel MJ. Vermeersch. Avaliação de Impacto na Prática. Washington: Banco Mundial, 2015.

30. Ivo AMS, Malta DC, Freitas MIF. Modos de pensar dos profissionais do Programa Academia da Saúde sobre saúde e doença e suas implicações nas ações de promoção de saúde. Physis. 2019;29(1):e290110. 